\title{
MONITORING THE EFFICACY OF ANTI-TB THERAPY BY USING THE QuantiFERON-TB Gold In tube test
}

\section{R. Markova*, R. Drenska*, Y. Todorova*, V. Terzieva* and D. Stefanova\#}

Research Excellence Award in Respiratory Infections, sponsored by Cellestis

*National Center of Infectious and Parasitic Diseases, Sofia 1504

\#University Hospital for Tuberculosis and Lung Diseases, Sofia 1601

WINNING ABSTRACT: Monitoring the efficacy of anti-TB therapy is crucial for the better control of the spread of MTB infection, but it is often difficult especially for patients without microbiological confirmation of the disease. IGRAs are novel indirect blood tests for M. tuberculosis infection and a promising marker of mycobacterial burden and disease activity.

To describe our experience with the use of the QuantiFERON-TB Gold In tube assay for monitoring of anti-TB therapy in patients with active disease 30 patients with active TB, all HIV-negative and BCG-vaccinated at birth, were studied before and after three and eight months of anti-TB therapy. The whole blood QFT G In tube assay was used to measure the MTB-specific IFN-gamma responses.

Before therapy $28 / 30$ patients had positive results in QFT G In tube (QFT) and two - indeterminate. Three months after initiation of treatment all subjects tested were found positive. On month eight a significant decrease of MTB-specific responses was found in all patients but only $17 / 30(56.7 \%)$ turned negative in QFT. Three of 13 patients with a positive response at the end of month eight, continued to have microbiological isolation and absence of clinical improvement (fig. 1, table 1).

On the basis of the results obtained, we concluded that the QFT Gold In tube assay could be used in routine clinical practice for monitoring anti-tuberculosis therapy. Further studies including larger number of patients and longer periods of observation are needed.

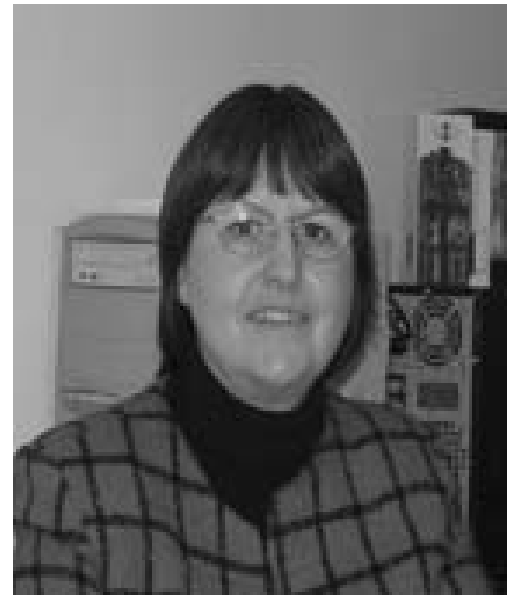

Roumiana Markova

Dept of Immunology and Allergy, National Center of Infectious and Parasitic Diseases, Sofia, Bulgaria

\section{SYNOPSIS OF MY JOB AND THE ROLE OF THE UNIT IN WHICH I WORK}

I work as an immunologist at the Dept of Immunology and Allergy of the National Center of Infectious and Parasitic Diseases (NCIPD) in Sofia, Bulgaria, where I am Head of the Laboratory of Mediators of Inflammation and Immunity. The NCIPD was founded in 1881 and, at present, is the leading institution in the Bulgarian healthcare system. It has the statute

STATEMENT OF INTEREST: This study was supported by the National Fund for Scientific Research, Ministry of Education and Science, Republic of Bulgaria (Grant L-1503/05). of a scientific organisation with a broad spectrum of activities. The NCIPD has a specific interest in infectious diseases, as well as allergic disorders and the development of new preventative and therapeutic vaccines and diagnostic tools against these diseases. Fundamental and applied research in the area of diagnostics, treatment and prophylaxis of infectious and parasitic diseases is carried out here.

The main field of scientific research of my team is immunology of tuberculosis (TB) infection and HIV/AIDS. We study the mechanisms of: immune dysregulation; cytokine production and expression; reconstitution of cell-mediated immune function during treatment; and modulation of specific and natural immunity.

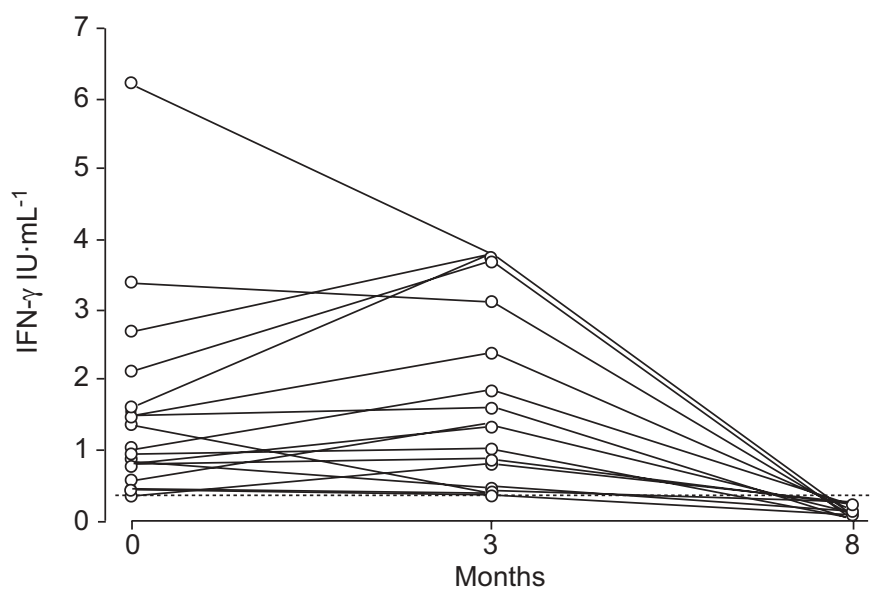

FIGURE 1. Changes of specific interferon (IFN)- $\gamma$ responses during antituberculosis (TB) therapy in $30 \mathrm{HIV}-/ \mathrm{TB}+$ patients. $\cdots \cdots \cdots \cdot 0.35 \mathrm{IU} \cdot \mathrm{mL}^{-1}$. 


\begin{tabular}{|c|c|c|c|c|c|c|c|}
\hline \multirow[t]{3}{*}{ TABLE 1} & \multicolumn{7}{|c|}{$\begin{array}{l}\text { Distribution of QuantiFERON-TB (QFT) In tube results in } 30 \text { TB+/HIV-patients before and } 3 \text { and } 8 \text { months after } \\
\text { specific anti-tuberculosis therapy }\end{array}$} \\
\hline & \multirow[t]{2}{*}{ Therapy } & \multicolumn{3}{|c|}{ Microbiologically confirmed $(n=10)$} & \multicolumn{3}{|c|}{ Microbiologically nonconfirmed $(n=20)$} \\
\hline & & QFT-G+ & QFT-G- & QFT-G+/- & QFT-G+ & QFT-G- & QFT-G+/- \\
\hline \multicolumn{2}{|c|}{ Before therapy } & 8 & 0 & 2 & 20 & 0 & 0 \\
\hline \multicolumn{2}{|l|}{3 months } & 10 & 0 & 0 & 20 & 0 & 0 \\
\hline \multicolumn{2}{|l|}{8 months } & 7 & 3 & 0 & 6 & 14 & 0 \\
\hline
\end{tabular}

We carry out follow-up of the natural course of infection and monitor the effects of specific anti-TB and/or anti-retroviral therapy (HAART) in patients with $\mathrm{TB}+, \mathrm{HIV}+$ and those coinfected with $\mathrm{HIV} / \mathrm{TB}$, by analysing various immune parameters such as antigen-specific CD4+ and CD8+ T-cell responses, T-helper (Th)1/Th2 cytokine production and expression, chemokine secretion and neutrophil function.

Another area of our work, which is jointly developed with the Laboratory of Respiratory Medicine of the Dept of Internal Medicine at the University of Rome "Tor Vergata" (Rome, Italy), is the identification and analysis of immunogenic Mycobacterium tuberculosis (MTB) peptides for the differential identification of subjects with active and latent TB infection in bacillus Calmette-Guerin vaccinated subjects.

My laboratory is well equipped and has been certified by ISO 17025 for its diagnostic activities. We were the first laboratory in Bulgaria and Europe to introduce the new interferon- $\gamma$ release assay (IGRA), QuantiFERON-TB (QFT; Cellestis Ltd, Melbourne, Australia) for the diagnosis of latent and active MTB infection. In addition, we routinely use the T-SPOT.TB assay. Both IGRAs proved useful in aiding the diagnosis of MTB infection, both in immunocompetent and immunocompromised individuals, and especially in those with HIV / AIDS. The in vitro immunodiagnosis of MTB infection is of great importance in patients with microscopy- and culture-negative results. Our studies demonstrated that CD4+ T-cell counts did not correlate with either antigen or mitogen stimulated interferon- $\gamma$ production and did not affect the results of IGRAs. The QFT test appeared to be more convenient and sensitive than the T-SPOT.TB test, with fewer indeterminate results in the immunosuppressed patients. We have also started using IGRAs for the monitoring of specific anti-TB therapy; I am responsible for the introduction and performance of new IGRAs under routine clinical conditions in Bulgaria. Our preliminary data suggest the possibility of using region of difference-1 immune responses as markers of efficacy during anti-TB treatment. Several studies have examined the effect of active TB treatment on specific interferon- $\gamma$ responses in patients with latent TB infection or active TB disease, and results have been inconsistent [1-5].

\section{HOW DOES MY RESEARCH FIT IN WITH THE OVERALL RESEARCH OF MY WORKING GROUP/RESEARCH TEAM?}

These studies fit very well into the main research area of our unit. We collaborate with many hospitals in Sofia and all over Bulgaria. The results from our studies are very useful for clinicians working in the field of tuberculosis and HIV infection and aid them in making the correct diagnosis and treatment. My team has been involved in many research projects supported by the National Scientific Fund at the Bulgarian Ministry of Education and Science. It also participates in international projects with scientific teams from Italy and India. We are involved in the development of clinically oriented research in the field of tuberculosis and are involved in the activities of the European Tuberculosis Network. As part of the postgraduate education programme of the National Center of Infectious and Parasitic Diseases, I teach $\mathrm{PhD}$ students and specialists in the field of immunology of infectious diseases, especially tuberculosis and HIV infection.

\section{REFERENCES}

1 Pathan AA, Wilkinson KA, Klenerman P, et al. Direct ex vivo analysis of antigen-specific IFN- $\gamma$ secreting CD4 T cells in Mycobacterium tuberculosis-infected individuals: associations with clinical disease state and effect of treatment. J Immunol 2001; 167: 5217-5225.

2 Carrara S, Vincenti D, Petrosillo N, Amicosante M, Girardi E, Goletti D. Use of a T cell-based assay for monitoring efficacy of antituberculosis therapy. Clin Infect Dis 2004; 38: 754-756.

3 Wilkinson KA, Kon OM, Newton SM, et al. Effect of treatment of latent tuberculosis infection on the $\mathrm{T}$ cell response to Mycobacterium tuberculosis antigens. J Infect Dis 2006; 193: 354-359.

4 Goletti D, Parracino M, Pasquale Butera O, et al. Isoniazid prophylaxis differently modulates T-cell responses to RD1epitopes in contacts recently exposed to Mycobacterium tuberculosis: a pilot study. Respir Res 2007; 8: 5.

5 Pai M, Joshi R, Bandyopadhyay M, et al. Sensitivity of a whole-blood interferon- $\gamma$ assay among patients with pulmonary tuberculosis and variations in $\mathrm{T}$-cell responses during anti-tuberculosis treatment. Infection 2007; 35: 98-103. 